\title{
An Experimental Study on Stress Sensitivity of Tight Sandstones with Different Microfractures
}

\author{
Xinli Zhao, ${ }^{1,2,3}$ Zhengming Yang, ${ }^{1,2,3}$ Zhiyuan Wang $\mathbb{D}^{4},{ }^{4}$ Wei Lin ${ }^{(D)},{ }^{1,2,3,5}$ \\ Shengchun Xiong, ${ }^{1,2,3}$ Yutian Luo, ${ }^{1,2,3}$ Zhongkun Niu, ${ }^{1,2,3}$ and Debin Xia ${ }^{1,2,3}$ \\ ${ }^{1}$ School of Engineering Science, University of Chinese Academy of Sciences, Beijing 100049, China \\ ${ }^{2}$ Institute of Porous Flow and Fluid Mechanics, Chinese Academy of Sciences, Langfang 065007, China \\ ${ }^{3}$ Department of Porous Flow \& Fluid Mechanics, Research Institute of Petroleum Exploration \& Development, \\ PetroChina Company Limited, Langfang 065007, China \\ ${ }^{4}$ Institute of Oceanography, Minjiang University, Fuzhou 350108, China \\ ${ }^{5}$ School of Geosciences, Yangtze University, Wuhan 430100, China
}

Correspondence should be addressed to Zhiyuan Wang; wangzhiyuan14@mails.ucas.edu.cn and Wei Lin; ucaslinwei@126.com Received 23 December 2019; Revised 8 May 2020; Accepted 18 May 2020; Published 29 May 2020

Academic Editor: Robert Černý

Copyright (c) 2020 Xinli Zhao et al. This is an open access article distributed under the Creative Commons Attribution License, which permits unrestricted use, distribution, and reproduction in any medium, provided the original work is properly cited.

\begin{abstract}
Aiming at the stress sensitivity problem of tight reservoirs with different microfractures, the cores of $\mathrm{H}$ oilfield and $\mathrm{J}$ oilfield with different microfractures were obtained through the fractures experiment, so as to study the change of gas permeability in tight sandstone core plug during the change of confining pressure. Besides, we use the nuclear magnetic resonance (NMR) spectra of the core before and after saturation to verify whether the core has been successfully fractured. Based on Terzaghi's effective stress principle, the permeability damage rate $(D)$ and the stress sensitivity coefficient $\left(S_{s}\right)$ are used to evaluate the stress sensitivity of the core, which show consistency in evaluating the stress sensitivity. At the same time, we have studied the petrological characteristics of tight sandstone in detail using thin section (TS) and scanning electron microscope (SEM). The results show that the existence of microfractures is the main factor for the high stress sensitivity of tight sandstone. In addition, because of the small throat of the tight reservoir core, the throat closes when the overlying stress increases. As a result, the tight sandstone pore size is greatly reduced and the permeability is gradually reduced. Therefore, in the development of tight reservoirs, we should not only consider the complex fracture network produced by fracturing, but also pay attention to the permanent damage of reservoirs caused by stress sensitivity.
\end{abstract}

\section{Introduction}

The tight reservoir has the typical characteristics of low porosity and low permeability [1]. Generally, tight oil and gas usually have no natural productivity, and their production capacities development often depends on large-scale hydraulic fracturing [2-6]. Fracture system is the main channel of fluid seepage in tight reservoir. Compared with pores, fractures have more unstable bracing structure and are more vulnerable to the change of effective stress. At the initial stage of production, most fractures are keeping open and act as the high-speed way for oil and gas transportation. With the continuous production and the decrease of formation pressure, some fractures are closing and the high- speed access to the production well is gradually cut off, resulting in a significant reduction in reservoir permeability and well production $[7,8]$. Therefore, it is of great theoretical and practical significance to investigate the stress sensitivity of fractured tight reservoirs.

It has been nearly 70 years since Fatt and Davis first observed permeability stress sensitivity, and stress sensitivity theory has been further developed and widely applied in the field of underground engineering [9]. Currently, petroleum engineers pay more attention to the tight reservoir stress sensitivity, with the development of unconventional oil and gas resources [10-12]. Some reports have also documented the stress sensitivity of fractured reservoirs. Jones discussed the stress sensitivity of fractured carbonate rocks and 
showed that the fracture permeability decreases logarithmically with the change of effective stress [13]. Chen et al. studied the stress sensitivity of shale and established the relationship between matrix permeability and effective stress and the relationship between fracture permeability and formation pressure [14]. Zhang et al. used the nuclear magnetic resonance (NMR) method to investigate the nonuniform change of pores and fractures with stress condition and divided it into two phases [15]. Although the predecessors have done a lot of work, the stress sensitivity of fractured tight sandstone reservoirs is not sufficiently studied, and there are many research studies focused on the evaluation of matrix sandstone reservoirs [16-21].

In this study, we employ the triaxial compression system to fabricate microfractures in tight sandstone core plugs, mimicking the hydraulic fracturing reservoirs. In addition, microfractures $(<0.1 \mathrm{~mm})$, which can only be characterized fully using optical and electron microscopy of core samples [22], are commonly observed in these tight oil sandstone reservoirs [23-25].

We double-check the microfracture formation by comparing the T2 spectra of saturated water cores before and after fracturing. In addition, we adopt Terzaghi's effective stress to evaluate the core plugs stress sensitivity and also compare and use the $D$ [26] and the $S_{s}$ [27] methods to evaluate the stress sensitivity of core plugs with microfractures. Finally, we analyzed the effects of reservoir petrological characteristics on stress sensitivity. This work contributes to the calculation and the evaluation of stress sensitivity of fractured tight sandstone reservoirs.

\section{Samples and Experimental Methods}

2.1. Tight Sandstone Samples. At present, the exploration and development of tight oil has made significant progress in the Ordos Basin, China. The Upper Triassic Yanchang Formation in the Ordos Basin is a multisource sedimentary basin that evolved in the lake basin in the Ordos Inland Depression. According to the characteristics of the sedimentary cycle, the Yanchang Formation is divided into 10 oil layer groups from the top to the bottom (Chang 1 to Chang 10) $[28,29]$. The Chang 8 and Chang 9 reservoirs in the $H$ oilfield and $J$ oilfield are the main layers of tight oil development. Among them, the Chang 8 reservoir tight sandstone type is dominated by feldspar lithic sandstone and lithic feldspar sandstone and contains a small amount of feldspar sandstone. The lithology of the Chang 9 reservoir is dominated by lithic feldspar sandstone, followed by feldspar sandstone and feldspar lithic sandstone [30]. A total of 14 cores were selected for this study, including 5 cores in the Chang 9 reservoir and 9 cores in the Chang 8 reservoir. According to the measured porosity/permeability data, i.e., the porosity between $6.23 \%$ and $17.00 \%$ with average porosity is $11.96 \%$. In addition, the permeability between $0.115 \times 10^{-3} \mu \mathrm{m}^{2}$ and $4.238 \times 10^{-3} \mu \mathrm{m}^{2}$, and the average permeability is $1.082 \times 10^{-3} \mu \mathrm{m}^{2}$. Hence, these core plugs are typical tight sandstone in target block. The detailed parameters information of the core plugs are shown in Table 1.
2.2. Experimental Methods. To mimic the tight sandstone of hydraulic fracturing reservoirs, a triaxial core compression test system is designed, as illustrated in Figure 1. The experimental system mainly consists of six parts: nitrogen cylinder $\left(\mathrm{N}_{2}\right)$, pressure stabilization device (voltage regulator), triaxial core holder, confining pressure pump (manual pump), axial pressure pump (Quizix pump), and micro flow meter. The nitrogen cylinder provides displacement pressure. The pressure stabilization device precisely regulates displacement pressure. The manual pump controls the confining pressure of cores. Quizix pump provides the axial pressure of cores. Triaxial core holder fixes and compresses cores. Micro flow meter included can measure the fluid flow rate with high accuracy.

The steps of artificial microfracture experiment in cores are as follows:

(1) First, the length, diameter, and dry weight of the core are measured.

(2) Close all valves and fix the prepared dry core in a triaxial core holder.

(3) After giving a certain confining pressure ( $3 \mathrm{MPa})$ and axial pressure, open the valve of the nitrogen bottle to allow the nitrogen to pass through the core stably. Observe the gas flow meter and record the data after stabilization. Then, calculate the permeability.

(4) Constantly increase the axial pressure. When the gas flow has reached a steady state, we begin to record the flow data and calculate the value of the permeability.

(5) By calculating the core permeability change rate of about $20 \%$ (Figure 2, point B), the experiment is stopped. At this point, close the valve of the nitrogen cylinder and remove the axial and confining pressures.

Figure 2 shows the relationship between gas permeability and axial pressure of typical cores; permeability change rate $R_{K}$ is calculated by

$$
R_{K}=\frac{K_{i}-K_{0}}{K_{0}} \times 100 \%,
$$

where $K_{0}$ is the gas permeability without the axial pressure, $\times 10^{-3} \mu \mathrm{m}^{2}$, and $K_{i}$ is the gas permeability under different axial pressures, $\times 10^{-3} \mu \mathrm{m}^{2}$.

It is found that when the axial pressure is less than $15 \mathrm{MPa}$, the core plug permeability change rate is negative and remains in a small range; this is because as the axial pressure increases, the tight sandstone pores are compressed and the permeability decreases slowly. When the axial pressure is $15-38 \mathrm{MPa}$, the permeability change rate gradually changes from negative to positive and has a greater change range; this indicates that microfractures are forming in the cores. Additionally, when the axial pressure is greater than $38 \mathrm{MPa}$, the increase in permeability change rate is very obvious; this is due to the formation of visible cracks in cores. Because in the process of volume fracturing, a large number of microfractures are formed in underground 
TABLE 1: Depth, porosity, and permeability of selected samples.

\begin{tabular}{|c|c|c|c|c|c|c|}
\hline Samples & Depth (m) & Subsection $(-)$ & $L(\mathrm{~cm})$ & $D(\mathrm{~cm})$ & Poro. (\%) & Perm. $(\mathrm{mD})$ \\
\hline HH56-7 & 2099.40 & Chang 9 & 6.533 & 2.532 & 17.00 & 3.619 \\
\hline HH56-9 & 2100.34 & Chang 9 & 6.869 & 2.532 & 16.80 & 4.238 \\
\hline HH56-15 & 2102.54 & Chang 9 & 6.987 & 2.533 & 14.45 & 1.062 \\
\hline HH56-16 & 2102.90 & Chang 9 & 6.969 & 2.532 & 15.02 & 1.154 \\
\hline НH56-18 & 2104.07 & Chang 9 & 7.013 & 2.533 & 16.64 & 2.104 \\
\hline HН190-4 & 2256.29 & Chang 8 & 6.864 & 2.526 & 15.74 & 0.447 \\
\hline HH190-25 & 2258.15 & Chang 8 & 7.155 & 2.525 & 10.86 & 0.459 \\
\hline HH107-4 & 2234.65 & Chang 8 & 7.139 & 2.533 & 14.43 & 0.202 \\
\hline НH17-17 & 2242.24 & Chang 8 & 6.601 & 2.532 & 8.53 & 0.113 \\
\hline $\mathrm{JH} 36-4$ & 1375.09 & Chang 8 & 7.260 & 2.534 & 7.95 & 0.359 \\
\hline JH36-13 & 1379.22 & Chang 8 & 6.503 & 2.531 & 9.47 & 0.710 \\
\hline JH23-17 & 1443.41 & Chang 8 & 6.995 & 2.532 & 6.39 & 0.235 \\
\hline JH23-24 & 1445.62 & Chang 8 & 6.848 & 2.533 & 7.89 & 0.330 \\
\hline JH23-28 & 1447.25 & Chang 8 & 6.747 & 2.534 & 6.23 & 0.115 \\
\hline
\end{tabular}

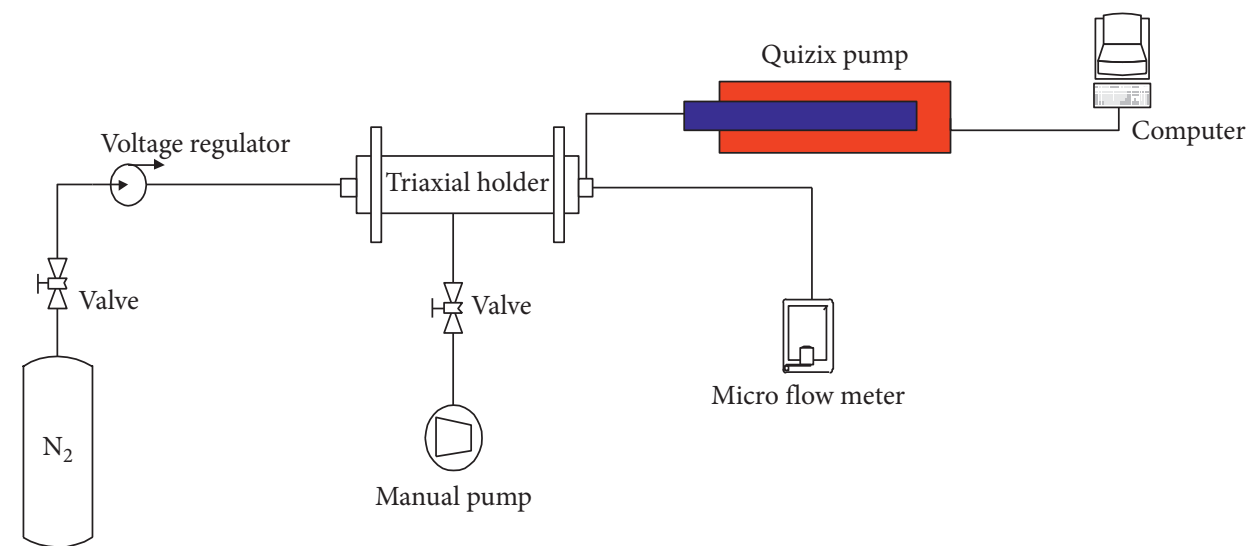

Figure 1: Core casting experiment diagram.

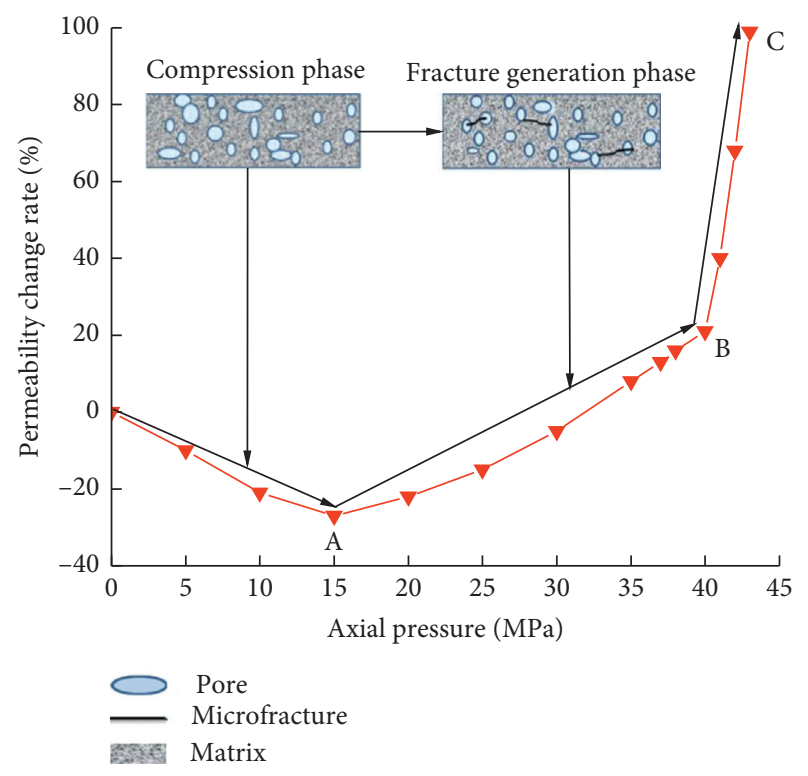

Figure 2: Diagram of permeability change with axial pressure.

reservoirs. The purpose of our work is to study the stress sensitivity of microfracture reservoirs, so we use point B (Figure 2) as the upper limit for fracturing.
Nuclear magnetic resonance (NMR) technology is to detect the presence of hydrogen nuclei in the target material (in the field of petroleum industry, we often study core plugs that are saturated with water or oil.), which results in the occurrence of NMR phenomenon. In this study, the NMR experiment was performed using the RecCore 2500 NMR core analyzer independently developed by the Institute of Porous Flow and Fluid Mechanics, CAS, China. The main test parameters are as follows: resonant frequency was $2.38 \mathrm{MHz}$, echo time was $0.25 \mathrm{~ms}$, and waiting time was $3000 \mathrm{~ms}$; echo number was 2048; experimental temperature was $25^{\circ} \mathrm{C}$. Three selected core samples were saturated with water before and after fracturing experiments, and then their $\mathrm{T} 2$ spectrums were tested.

The stress sensitivity of reservoir is caused by the deformation of tight sandstone and the change of permeability [31]. The change of reservoir permeability is the result of the change of effective stress, so the study of reservoir stress sensitivity is mainly about the relationship between permeability and effective stress $[32,33]$. The change of effective stress is realized by controlling the change of confining pressure while the injection fluid pressure remains unchanged. In the specific experiment process, we refer to the oil industry standard SY/T 5358-2010. 
In addition, we use the Terzaghi effective stress to evaluate the core stress sensitivity. The calculation formula is as follows [10]:

$$
\sigma_{\text {eff }}^{p}=\sigma-p_{\mathrm{p}}
$$

where $\sigma_{\text {eff }}^{p}$ is the effective stress, $\mathrm{MPa} \sigma \sigma$ is the overburden pressure, $\mathrm{MPa}$; and $p_{\mathrm{p}}$ is the pore pressure, $\mathrm{MPa}$.

In this paper, the effective stress in evaluating the stress sensitivity of tight sandstone refers to the Terzaghi effective stress. The tight sandstone permeability calculation formula under any internal pressure $p$ is as follows [34]:

$$
K=K_{\mathrm{f}} e^{-b\left(p_{\mathrm{i}}-p\right)},
$$

where $K$ is the tight sandstone permeability, $10^{-3} \mu \mathrm{m}^{2} ; K_{\mathrm{f}}$ is the tight sandstone permeability under the original formation pressure, $\times 10^{-3} \mu \mathrm{m}^{2}$; $b$ is the stress sensitivity constant, $\mathrm{MPa}^{-1} ; p_{\mathrm{i}}$ is the original formation pressure, $\mathrm{MPa}$; and $p$ is the internal pressure of the tight sandstone, MPa.

\section{Results}

3.1. Core Fracture Test Results. We used a triaxial compression test system to perform microfractures-forming experiments on cores with different permeability. The result is as follows. It can be seen from Figure 3 that a total of three stages are experienced in the process of continuously increasing the axial pressure. When the axial pressure starts to increase, the permeability decreases slightly. Then, as the axial pressure increases, the permeability begins to increase, and then the inflection point occurs. At this time, the core has micro microfractures that cannot be observed by the naked eye. As the permeability change rate continues to increase, the more microfractures are created in the core. In order to verify whether the core is fractured after the microfractures are formed, we also use the nuclear magnetic resonance technique to test the $\mathrm{T} 2$ spectrum of the tight sandstone sample under saturated water before and after the microfractures. From Figure 4, we can see that the T2 spectrum of the core in the saturated water state after the microfractures is higher than the T2 spectrum in the saturated water state before the microfractures formation. The higher T2 spectrum is partly due to the saturation of the fractures in the core. This also indicates that the core will have microfractures after the microfractures are formed. The proportion of the microfractures in the three cores after the microfractures is $11.12 \%, 15.67 \%$, and $12.33 \%$, respectively. At the same time, NMR testing indicates that core microfractures create or open microfractures. If a large-scale fracture occurs, a third peak appears at the right end of the NMR spectrum.

Besides, different cores have different fracturing pressures. The fracturing pressure of all cores is between 20 and $30 \mathrm{MPa}$. Among them, the HH56-18 core has the lowest fracturing pressure of $20 \mathrm{MPa}$ and the HH190-25 core has the largest fracturing pressure of $30 \mathrm{MPa}$. Moreover, the HH190-25 core has the highest rate of change in permeability as the axial pressure increases. This may be the result of the development of high-angle microfractures in the core.

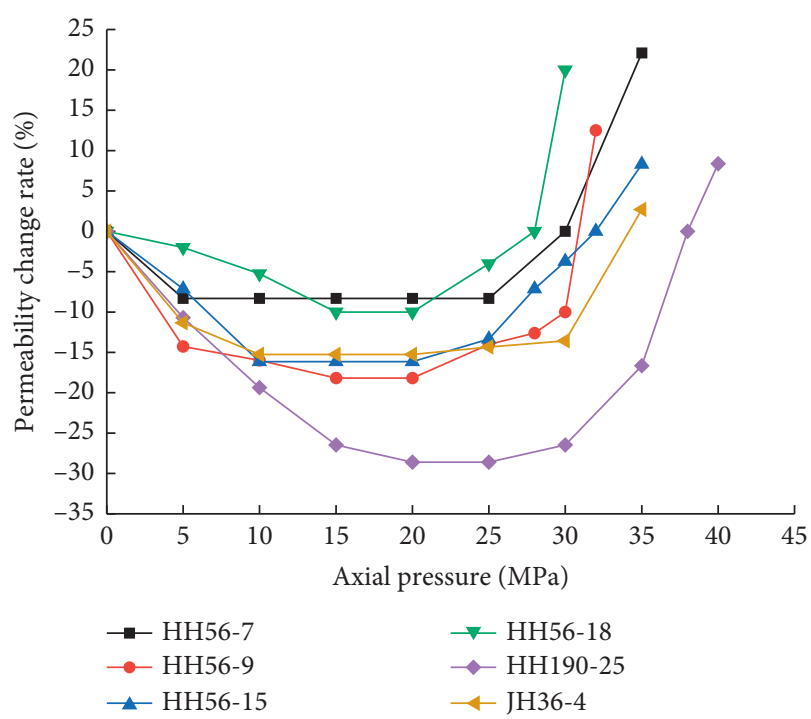

FIgURE 3: Permeability change rate with axial pressure curve.

3.2. Stress Sensitivity. Three typical cores were selected and stress sensitivity experiments were performed before and after microfractures formation (see Figure 5). The stress sensitivity curve is divided into two phases. The effective stress is steeper before $20 \mathrm{MPa}$, but as the effective stress increases, the curve becomes slow. In addition, the sensitivity curves of the core before and after the microfractures are not coincident, and the stress sensitivity after the core is stronger than before the microfractures are created. As the permeability decreases, the stress sensitivity of the core increases. During the loading process, there is a good power relationship between the change in permeability and the effective stress $\left(R^{2}>0.9\right)$.

There is stress-sensitive hysteresis in the experimental core. After the effective stress is released, the core plugs permeability cannot be completely recovered, and there is a certain degree of permeability loss. When the effective stress exceeds a certain value, the change curve of the permeability retention rate of the pressurization phase and the pressure relief phase coincide. This indicates that the effective stress increases early, the core undergoes structural deformation and body deformation, and the permeability changes significantly. However, when the effective stress exceeds a certain value, the core pore structure changes tend to be stable, and the core deformation is the body deformation. At the same time, the change in permeability has also become stable. This phenomenon is consistent with that studied by Cao et al. [11].

\section{Discussion}

4.1. Evaluation of Stress Sensitivity. There are many studies on the evaluation methods of stress sensitivity. In China, scholars usually use the industry standard SY/T 5358-2010 to evaluate the stress sensitivity of tight sandstones [26, 35]. It is believed that tight sandstones are generally strongly stress sensitive. In this study, we also use this evaluation method and combine it with the $S_{\mathrm{s}}$ to determine the stress sensitivity 


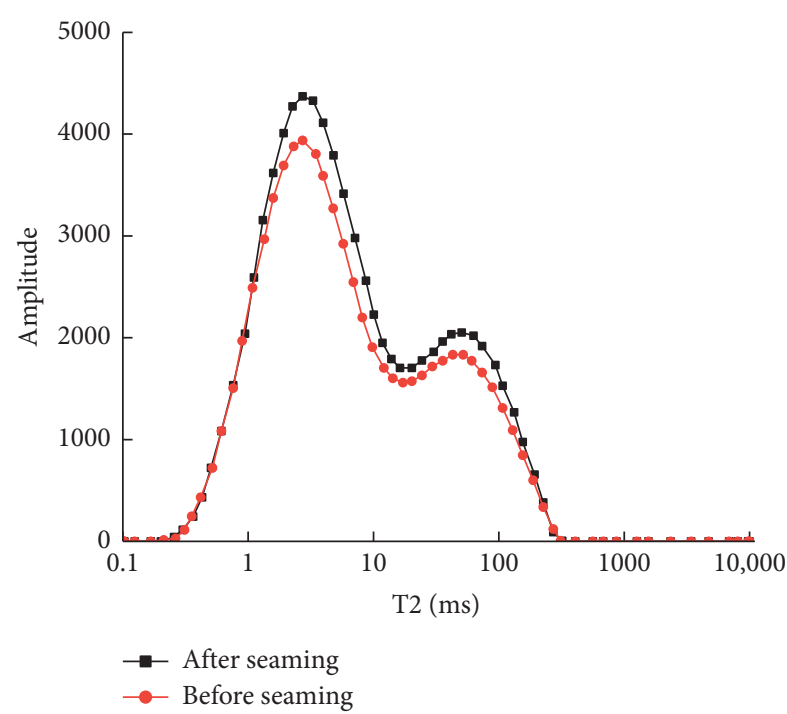

(a)

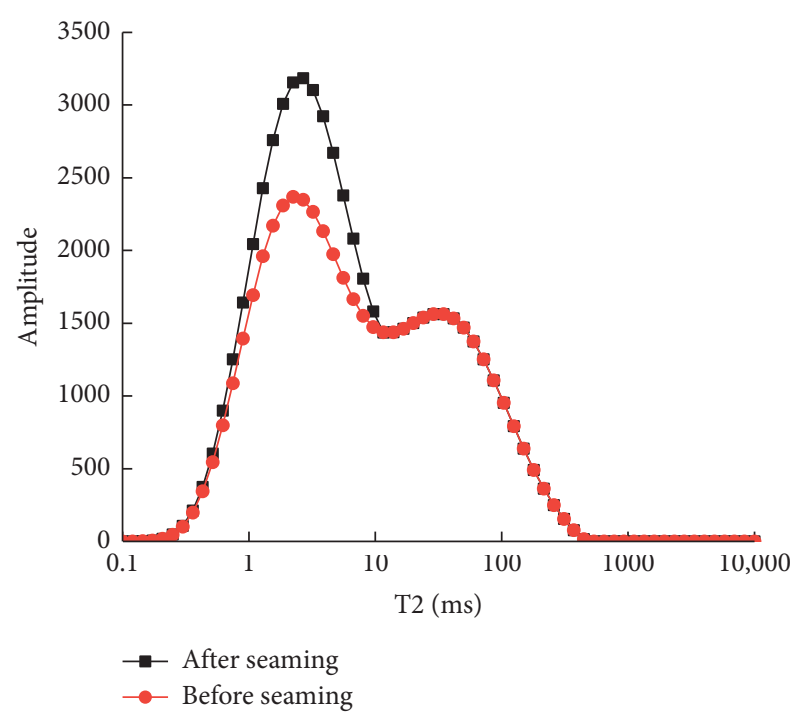

(b)

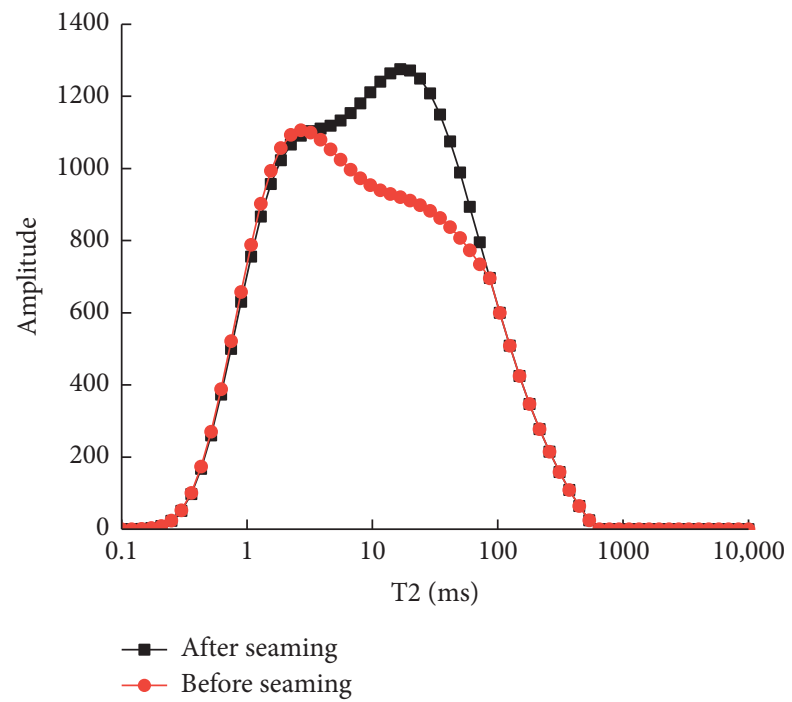

(c)

FIGURE 4: Nuclear magnetic resonance T2 spectrum of tight sandstone samples before and after fracturing. (a) HH56-15 core sample. (b) HH190-25 core sample. (c) JH36-4 core sample.

of reservoir tight sandstones to study the stress sensitivity of tight sandstone. First, we use equation (4) to calculate the rate of change of permeability under different effective stresses during the effective stress increase process (SY/T 5358-2010):

$$
D=\frac{K_{m}-K_{n}}{K_{m}} \times 100 \%,
$$

where $D$ is the rate of change of permeability under different effective stresses during the effective stress increase process, $\% ; K_{m}$ is the core permeability under the initial effective stress, $10^{-3} \mu \mathrm{m}^{2}$; and $K_{n}$ is the core permeability under different effective stresses during the effective stress increase process, $10^{-3} \mu \mathrm{m}^{2}$.

In addition, we also use the stress sensitivity coefficient $S_{\mathrm{s}}$ proposed in [27] to classify the stress sensitivity of reservoir rocks. The calculation method of stress sensitivity coefficient is as follows:

$$
\left(\frac{K}{K_{\text {ref }}}\right)^{1 / 3}=1-S_{\mathrm{s}} \ln \left(\frac{\sigma_{\text {re }}}{\sigma_{\text {ref }}}\right),
$$

where $K$ is the core permeability, $10^{-3} \mu \mathrm{m}^{2} ; K_{\text {ref }}$ is the permeability under reference effective stress $\sigma_{\text {ref }}, 10^{-3} \mu \mathrm{m}^{2} ; S_{\mathrm{s}}$ is the stress sensitivity coefficient of the logarithmic model, dimensionless; $\sigma_{\text {re }}$ is the effective stress, $\mathrm{MPa}$; and $\sigma_{r e f}$ is the reference effective stress, $\mathrm{MPa}$.

We use the $S_{\mathrm{s}}$ to classify the stress sensitivity of tight sandstones, where $S_{s}>0.40$ is the strong stress sensitivity; $S_{s}<0.25$ is the weak stress sensitivity; and $0.25 \leq S_{s} \leq 0.4$ is the medium stress sensitivity [27].

The calculation and evaluation results are shown in Table 2. It can be seen from Table 2 that both $D$ and $S_{s}$ 


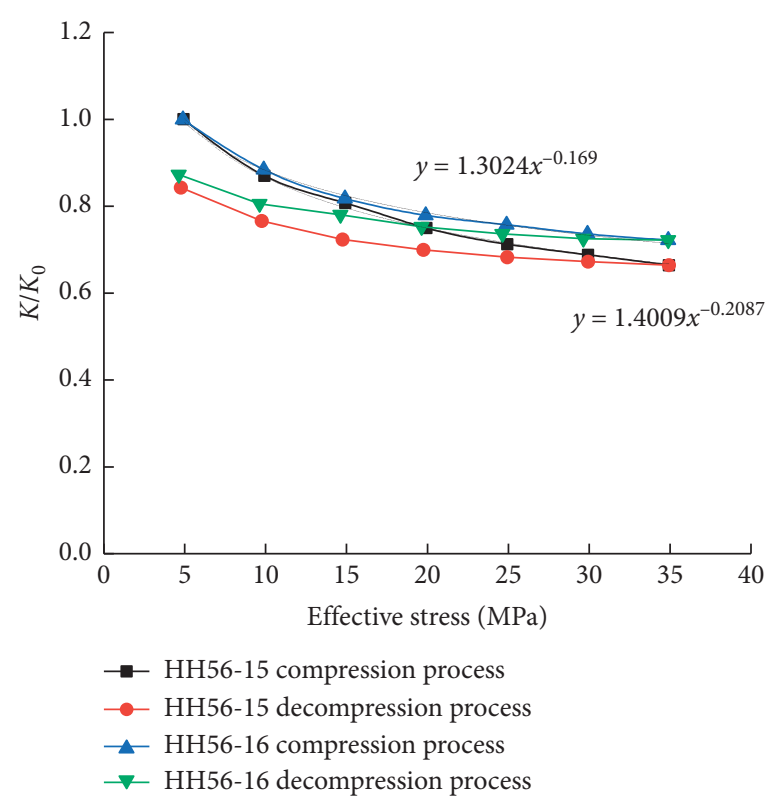

(a)

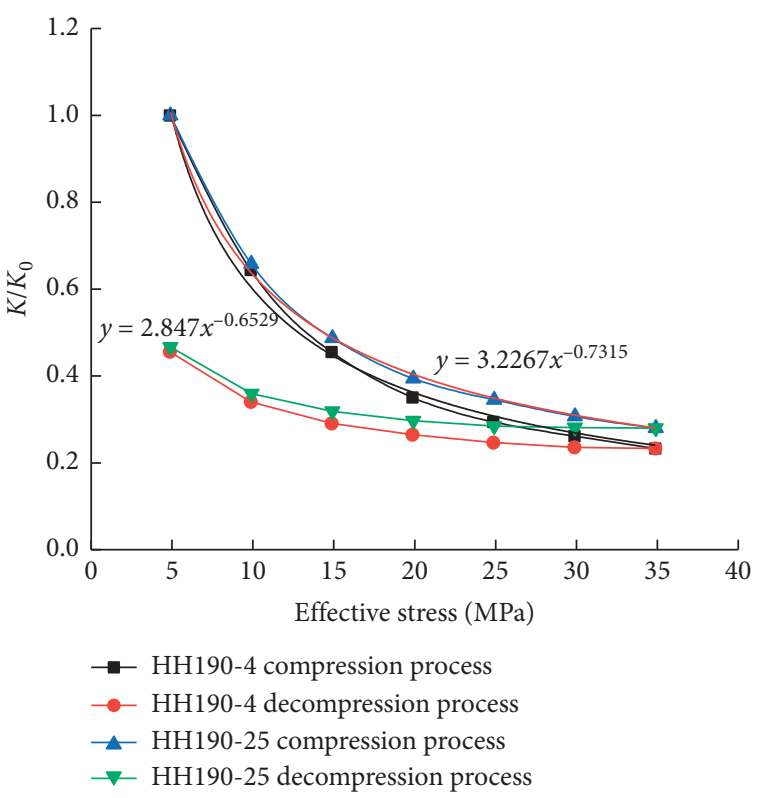

(b)

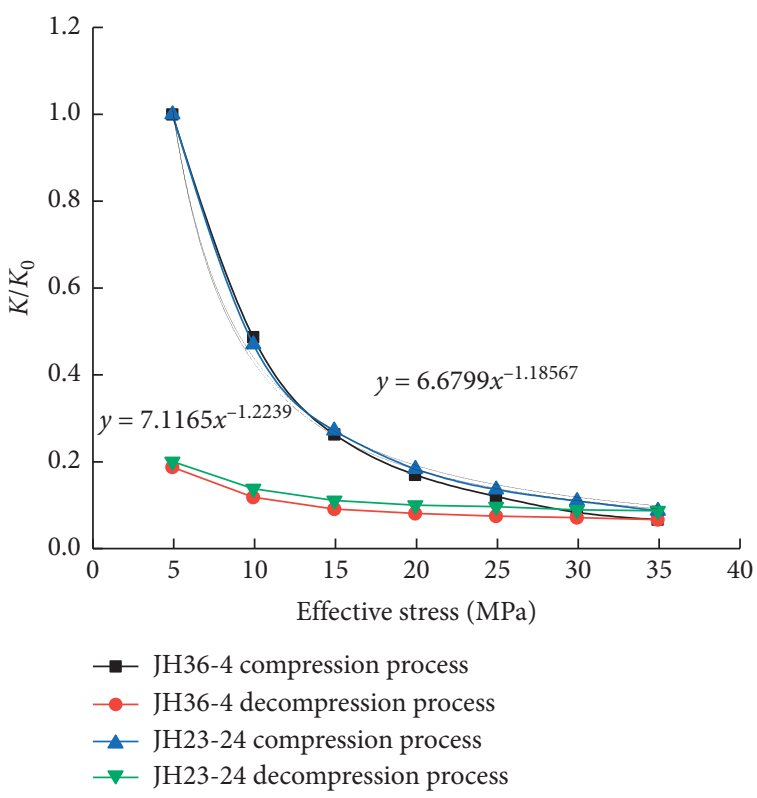

(c)

Figure 5: Permeability retention rate of cores at different effective pressures.

methods can be used to assess stress sensitivity. When the permeability of the core plug is less than $1 \times 10^{-3} \mu \mathrm{m}^{2}$, the two methods can have good consistency. When the core permeability is more than $1 \times 10^{-3} \mu \mathrm{m}^{2}$, the $S_{\mathrm{s}}$ method shows that the core has medium and weak sensitivity. But $D$ method shows that there is weak stress sensitivity in core. Although the two methods have different evaluation results in different permeability intervals, the industry standard only uses the maximum permeability and minimum permeability values when evaluating stress sensitivity. The $S_{\mathrm{s}}$ method is obtained by fitting all experimental data. A tight sandstone sample corresponds to one $S_{\mathrm{s}}$, which is holistic and unique, and the evaluation is more objective.
Furthermore, we can see from Figure 6 that when the core plug permeability is less than $1 \times 10^{-3} \mu \mathrm{m}^{2}$ and the porosity is less than $10 \%$, the core exhibits strong stress sensitivity. This is consistent with the results of previous studies on tight sandstones showing strong stress sensitivity.

\subsection{Effect of Petrological Characteristics of Reservoir on Stress} Sensitivity. Deformation under effective stress is the main factor for stress sensitivity of core [36]. It can be seen from Figure 7 that the pore system of the study block mainly includes intergranular pore space, intragranular pore space, and microfractures. The deformation is influenced by tight 
TABLE 2: Core stress sensitivity evaluation results.

\begin{tabular}{lccccc}
\hline Samples & $b$ & $D$ & Results & $S$ & Results \\
\hline HH56-7 & 0.0121 & 23.56 & Weak & 0.0801 & Medium \\
HH56-9 & 0.0130 & 18.63 & Weak & Weak \\
HH56-15 & 0.0101 & 33.60 & Moderate & 0.1217 & Medium \\
HH56-16 & 0.0030 & 27.81 & Weak & 0.1066 & Medium \\
HH56-18 & 0.0157 & 25.06 & Weak & 0.4604 & Medium \\
HH190-4 & 0.0135 & 76.70 & Strong & 0.4115 & Strong \\
HH190-25 & 0.0170 & 71.98 & Strong & 0.4859 & Medium \\
HH107-4 & 0.0193 & 79.60 & Strong & 0.7117 & Strong \\
HH17-17 & 0.0297 & 83.23 & Strong & 0.6314 & Strong \\
JH36-4 & 0.0244 & 93.38 & Strong & 0.6767 & Strong \\
JH36-13 & 0.0271 & Strong & 0.6587 & Strong \\
JH23-17 & 0.0261 & Strong & 0.7256 & Strong \\
JH23-24 & 0.0306 & 91.33 & & 0.0801 \\
JH23-28 & 0.0121 & 93.83 & & Strong \\
\hline
\end{tabular}

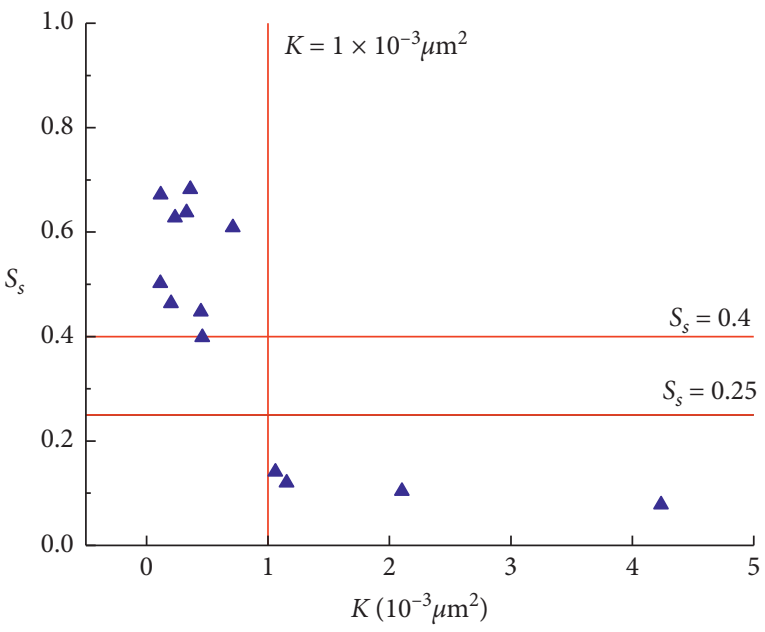

(a)

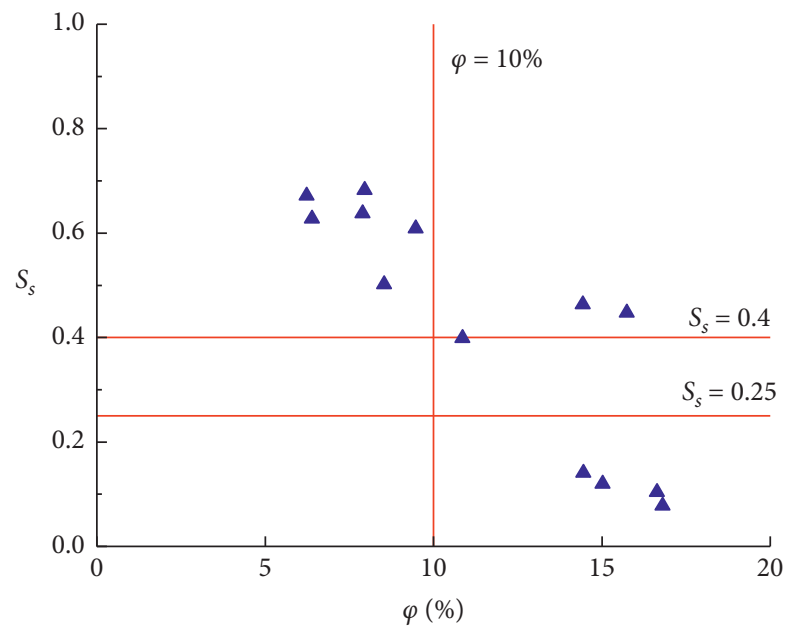

(b)

FIGURE 6: Core sample stress sensitivity coefficient and permeability and porosity intersection diagram. (a) Relationship between permeability $(K)$ and stress sensitivity coefficient $\left(S_{\mathrm{s}}\right)$. (b) Relationship between porosity $(\varphi)$ and stress sensitivity coefficient $\left(S_{\mathrm{s}}\right)$.

sandstone composition, contact mode of tight sandstone particles, and pore type. Therefore, this part compares the control of stress sensitivity with tight sandstone composition, tight sandstone particle contact mode, and pore type of reservoirs in the two oilfields ( $\mathrm{H}$ and J oilfields). Firstly, microfractures of different scales are developed in reservoir cores of $\mathrm{H}$ and J oilfields (Figures 7(b) and 7(e)). This is the main reason for the strong stress sensitivity of reservoirs (Table 2). The tight sandstones in $\mathrm{H}$ oilfield are mainly composed of quartz, feldspar, and cuttings, of which the quartz content is less than $50 \%$. The contact between particles is mainly linear contact and concave-convex contact. The proportion of fine and medium-sized detrital particles is more than $90 \%$, and their respective proportions are roughly the same. At same time, intergranular pore in core is not well developed and its connectivity is poor. Some tight sandstones have intragranular dissolved pore with less porosity (Figure $7(\mathrm{a})$ ). The pore size distribution ranges from $0.02 \mathrm{~mm}$ to $0.21 \mathrm{~mm}$. In addition, based on the scanning electron microscopy (SEM) analysis of reservoir rock samples in $\mathrm{H}$ oilfield, it is found that parts of the grain margins are eroded and there are a few intergranular dissolution pore and dissolution micropore (Figure $7(\mathrm{c})$ ). Intergranular fillings are mostly composed of authigenic kaolinite, illite, illite-montmorillonite mixtures, and mud (Figure 7(c)). Moreover, the intercrystalline micropore of the fillings is less, and the inter-granular pore of the tight sandstones is not developed. The face percentage is less than $1 \%$, and the average clay content is about $15 \%$. The clay minerals kaolinite and illite distributed in J oilfield can make the porosity of sandstone decrease and the physical properties of reservoir deteriorate. The contact relationship between particles is mainly point and line, where pore size ranges from $0.01 \mathrm{~mm}$ to $0.08 \mathrm{~mm}$. In addition, the segregation of reservoir rock particles in J oilfield was found to be moderate by scanning electron microscopy (SEM) (Figure 7(f)). Intergranular fillings are mostly illite, illitemontmorillonite mixed layer, kaolinite, and authigenic quartz. Because the intergranular pore is not developed, the facial porosity is low. Quartz in some tight sandstones 


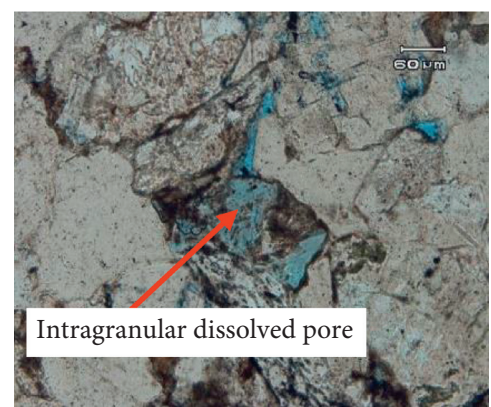

(a)

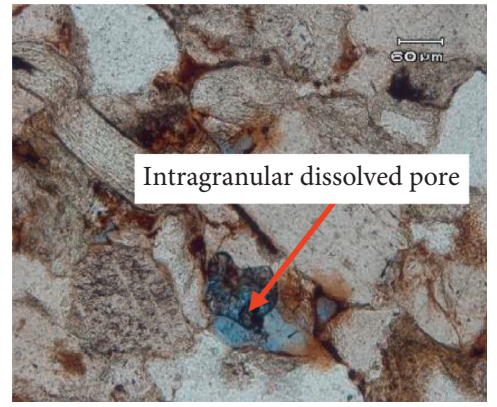

(d)

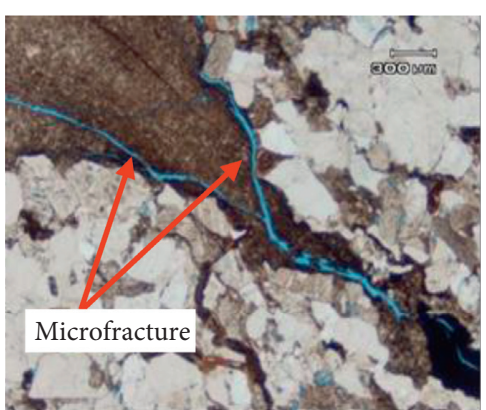

(b)

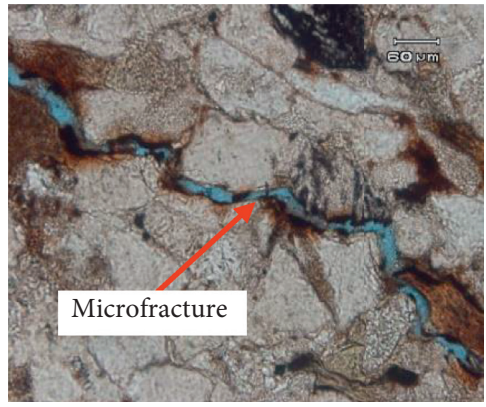

(e)

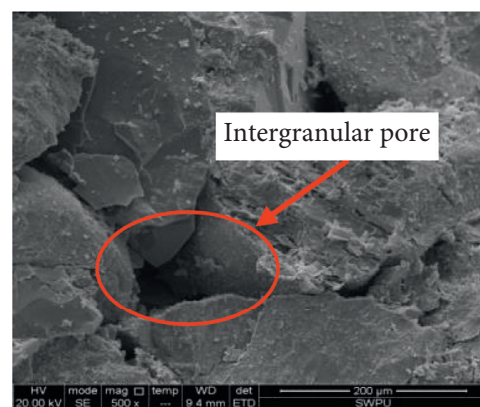

(c)

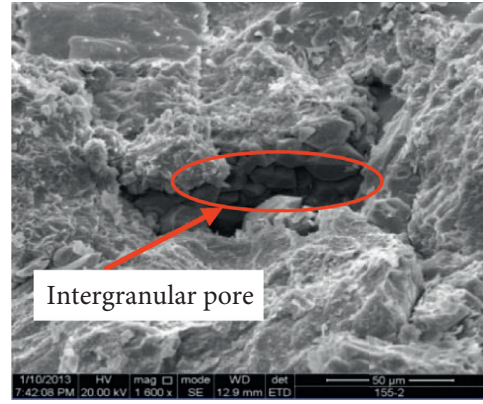

(f)

FIgURE 7: TS and SEM images of $\mathrm{H}$ and J oilfields. $(\mathrm{a}, \mathrm{b})$ Casting thin section (TS) images of $\mathrm{H}$ oilfield reservoirs. (c) Scanning electron microscopy (SEM) image of reservoir cores in $\mathrm{H}$ oilfield. (d, e) TS images of J oilfield reservoirs. (f) SEM image of reservoir cores in J oilfield.

increased slightly and feldspar was dissolved in some tight sandstones. There are fewer intergranular pore and intergranular dissolved pore. The average clay content is about $20 \%$. Tight sandstone has a small throat and is very sensitive to pressure $[37,38]$. When the overlying stress is slightly increased, the small throat is extremely vulnerable to pressure closure, which results in a large reduction in pore throat size and a rapid decrease in permeability.

\section{Conclusions}

In this work, we designed a core-made fractures experimental system and obtained cores with different microfractures development. Stress sensitivity of different microfracture development cores was studied by means of fixed confining pressure and stress sensitivity of pore pressure. We conclude that tight sandstone cores have strong stress sensitivity. The more developed the microfracture is, the stronger the stress sensitivity is. At the same time, we used $D$ and $S_{\mathrm{s}}$ to evaluate the sensitivity of the tight sandstone core plugs stress; only when the permeability of core is less than $1 \times 10^{-3} \mu \mathrm{m}^{2}$ can the two methods have good consistency. Both methods suggest that tight sandstones have strong stress sensitivity. But when the core permeability is more than $1 \times 10^{-3} \mu \mathrm{m}^{2}$, the $S_{\mathrm{s}}$ method shows that the core has medium and weak sensitivity. In addition, SEM and thin section (TS) tests suggest that microfractures are well developed in tight sandstones of $\mathrm{H}$ and $\mathrm{J}$ reservoirs. Through the analysis of petrological characteristics, it is considered that the core particle contact relationship, pore throat size, and pore throat type of tight oil reservoir have a certain degree of influence on stress sensitivity.

\section{Data Availability}

The data used to support the findings of this study are available from the corresponding author upon request.

\section{Disclosure}

Zhiyuan Wang is the co-first author.

\section{Conflicts of Interest}

All authors declare that there are no conflicts of interest regarding the publication of this article.

\section{Acknowledgments}

The authors gratefully acknowledge the financial support from the National Science and Technology Major Project of China (Grants nos. 2017ZX05013-001 and 2017ZX05069003), the National Science Foundation (Grant no. 1724469), and the Scientific Research Foundation for the Ph.D., Minjiang University (no. MJY19032).

\section{References}

[1] J. Lai, G. Wang, Z. Wang et al., "A review on pore structure characterization in tight sandstones," Earth-Science Reviews, vol. 177, pp. 436-457, 2018.

[2] L. Wang and X. Wang, "Modelling of pressure transient behaviour for fractured gas wells under stress-sensitive and slippage effects," International Journal of Oil, Gas and Coal Technology, vol. 11, no. 1, pp. 18-38, 2016. 
[3] X. Zhao, Z. Yang, W. Lin, S. Xiong, and Y. Wei, "Characteristics of microscopic pore-throat structure of tight oil reservoirs in Sichuan Basin measured by rate-controlled mercury injection," Open Physics, vol. 16, no. 1, pp. 675-684, 2018.

[4] L. Li, J. Tan, D. A. Wood et al., "A review of the current status of induced seismicity monitoring for hydraulic fracturing in unconventional tight oil and gas reservoirs," Fuel, vol. 242, pp. 195-210, 2019.

[5] Z. Ren, R. Yan, X. Huang et al., "The transient pressure behavior model of multiple horizontal wells with complex fracture networks in tight oil reservoir," Journal of Petroleum Science and Engineering, vol. 173, pp. 650-665, 2019.

[6] H. Yu, Z. Yang, L. Luo et al., "Application of cumulative-insitu-injection-production technology to supplement hydrocarbon recovery among fractured tight oil reservoirs: a case study in Changqing oilfield, China," Fuel, vol. 242, pp. 804$818,2019$.

[7] B. Liang, H. Jiang, J. Li et al., "Flow in multi-scale discrete fracture networks with stress sensitivity," Journal of Natural Gas Science and Engineering, vol. 35, pp. 851-859, 2016.

[8] Y. Liu, P. Yu, and Z. Ding, "Research on stress-sensitivity of fractured porous media," Journal of Petroleum Science and Engineering, vol. 171, pp. 879-889, 2018.

[9] I. Fatt and D. H. Davis, "Reduction in permeability with overburden pressure," Journal of Petroleum Technology, vol. 4, no. 12, p. 16, 1952.

[10] H. Dou, H. Zhang, S. Yao et al., "Measurement and evaluation of the stress sensitivity in tight reservoirs," Petroleum Exploration and Development, vol. 43, no. 6, pp. 1116-1123, 2016.

[11] N. Cao and G. Lei, "Stress sensitivity of tight reservoirs during pressure loading and unloading process," Petroleum Exploration and Development, vol. 46, no. 1, pp. 138-144, 2019.

[12] Q. H. Xiao, X. L. Zhao, W. Lin, X. L. Huang, and Z. B. Song, "Application of NMR to test sandstone stress sensitivity of the Dongfang X gas field, China," IEEE Access, vol. 7, no. 1, pp. 95200-95211, 2019.

[13] S. C. Jones, "A technique for faster pulse-decay permeability measurements in tight rocks," SPE Formation Evaluation, vol. 12, no. 1, pp. 19-26, 1997.

[14] D. Chen, Z. Pan, and Z. Ye, "Dependence of gas shale fracture permeability on effective stress and reservoir pressure: model match and insights," Fuel, vol. 139, pp. 383-392, 2015.

[15] J. Zhang, C. Wei, W. Ju et al., "Stress sensitivity characterization and heterogeneous variation of the pore-fracture system in middle-high rank coals reservoir based on NMR experiments," Fuel, vol. 238, pp. 331-344, 2019.

[16] K. Terzaghi, "Die berechnung der durchlaeassigkeitsziffer des tones aus dem verlauf der hydrodynamoschen spannungserscheinungen," Sitzungsberichte der Kaiserlichen Akademie der Wissenschaften, vol. 132, pp. 125-138, 1923.

[17] Y. Xu, Y. Wang, H. Yuan, D. Zhang, F. Agostini, and F. Skoczylas, "Pore structure characterization of tight sandstone from Sbaa Basin, Algeria: investigations using multiple fluid invasion methods," Journal of Natural Gas Science and Engineering, vol. 59, pp. 414-426, 2018.

[18] Y. Yang, W. Zhang, Y. Gao et al., "Influence of stress sensitivity on microscopic pore structure and fluid flow in porous media," Journal of Natural Gas Science and Engineering, vol. 36, pp. 20-31, 2016.

[19] B. Liu, Y. Q. Yang, J. T. Li, J. H. Li, and X. F. Fu, "Stress sensitivity of tight reservoirs and its effect on oil saturation: a case study of lower cretaceous tight clastic reservoirs in the
Hailar Basin, Northeast China," Journal of Petroleum Science and Engineering, vol. 184, Article ID 106484, 2019.

[20] T. Yin, P. Wang, J. Yang, and X. Li, "Mechanical behaviors and damage constitutive model of thermally treated sandstone under impact loading," IEEE Access, vol. 6, pp. 72047-72062, 2018.

[21] J. Zhang, C. Wei, W. Ju et al., "Stress sensitivity characterization and heterogeneous variation of the pore-fracture system in middle-high rank coals reservoir based on NMR experiments," Fuel, vol. 238, pp. 331-344, 2019.

[22] M. H. Anders, S. E. Laubach, and C. H. Scholz, "Microfractures: a review," Journal of Structural Geology, vol. 69, no. Part B, pp. 377-394, 2014.

[23] L. Zeng, H. Su, X. Tang, Y. Peng, and L. Gong, "Fractured tight sandstone oil and gas reservoirs: a new play type in the Dongpu depression, Bohai Bay Basin, China," AAPG Bulletin, vol. 97, no. 3, pp. 363-377, 2013.

[24] J. N. Hooker, S. E. Laubach, and R. Marrett, “A universal power-law scaling exponent for fracture apertures in sandstone," Geological Society of America Bulletin, vol. 126, no. 910, pp. 1340-1362, 2014.

[25] J. Lai, G. Wang, Z. Fan et al., "Fracture detection in oil-based drilling mud using a combination of borehole image and sonic logs," Marine and Petroleum Geology, vol. 84, pp. 195-214, 2017.

[26] National Energy Administration, Formation Damage Evaluation by Flow Test: SY/T 5358-2010, Petroleum Industry Press, Beijing, China, 2010.

[27] W. Xiao, T. Li, M. Li, J. Zhao, L. Zheng, and L. Li, "Evaluation of the stress sensitivity in tight reservoirs," Petroleum Exploration and Development, vol. 43, no. 1, pp. 115-123, 2016.

[28] J. Lai, G. Wang, Z. Fan, Z. Zhou, J. Chen, and S. Wang, "Fractal analysis of tight shaly sandstones using nuclear magnetic resonance measurements," AAPG Bulletin, vol. 102, no. 2, pp. 175-193, 2018.

[29] J. Lai, G. Wang, Y. Ran, Z. Zhou, and Y. Cui, "Impact of diagenesis on the reservoir quality of tight oil sandstones: the case of upper Triassic Yanchang Formation Chang 7 oil layers in Ordos Basin, China," Journal of Petroleum Science and Engineering, vol. 145, pp. 54-65, 2016.

[30] P. Li, W. Sun, J. Yan, R. Huang, and H. Huang, "Microscopic pore structure of Chang 63 reservoir in Huaqing oilfield, Ordos Basin, China and its effect on water flooding characteristics," Journal of Petroleum Exploration and Production Technology, vol. 8, no. 4, pp. 1099-1112, 2018.

[31] Y. Meng, Z. Li, and F. Lai, "Experimental study on porosity and permeability of anthracite coal under different stresses," Journal of Petroleum Science and Engineering, vol. 133, pp. 810-817, 2015.

[32] H. H. Liu, H. Chen, Y. Han, S. L. Eichmann, and A. Gupta, "On the relationship between effective permeability and stress for unconventional rocks: analytical estimates from laboratory measurements," Journal of Natural Gas Science and Engineering, vol. 56, pp. 408-413, 2018.

[33] Z. H. Liu, B. J. Bai, Z. Y. Zhang, J. Tang, S. P. Zeng, and X. G. Li, "Experimental study on the influence of slickwater on shale permeability," IOP Conference Series: Earth and Environmental Science, vol. 113, p. 12225, 2018.

[34] Y. Gu, W. Ding, M. Yin et al., "Study on pressure sensitivity of tight sandstone and its influence on reservoir characteristics," Energy Sources, Part A: Recovery, Utilization, and Environmental Effects, vol. 40, no. 22, pp. 2671-2677, 2018.

[35] B. Y. Wang, Y. Qing, J. Shen, and G. Wang, "Study on stress sensitivity of lignite reservoir under salinity and $\mathrm{pH}$ 
composite system," Energy Exploration \& Exploitation, vol. 36, no. 3, pp. 464-487, 2017.

[36] Z. H. Wu, Y. J. Zuo, S. Y. Wang, J. B. Sunwen, and L. L. Liu, "Experimental study on the stress sensitivity and influence factors of shale under varying stress," Shock and Vibration, vol. 2018, Article ID 3616942, 9 pages, 2018.

[37] X. L. Zhao, Z. M. Yang, W. Lin et al., "Study on pore structures of tight sandstone reservoirs based on nitrogen adsorption, high-pressure mercury intrusion, and rate-controlled mercury intrusion," Journal of Energy Resources Technology, vol. 141, no. 11, 2019.

[38] X. Zhao, Z. Yang, W. Lin et al., "Fractal study on pore structure of tight sandstone based on full-scale map," International Journal of Oil, Gas and Coal Technology, vol. 22, no. 2, pp. 123-139, 2019. 